\title{
Measurement of the Point Spread Function for Low-Loss Inelastic Scattering
}

R.F. Egerton ${ }^{1 *}$, A.M. Blackburn ${ }^{2}$, R.A. Herring ${ }^{3}$, L. $\mathrm{Wu}^{4}$ and Y. Zhu ${ }^{4}$

1. Physics Department, University of Alberta, Edmonton, Canada T6G 2 E1.

2. CAMTEC, University of Victoria, Canada V8W 2 Y2.

3. Microscopy Facility, University of Victoria, Canada V8W 2 Y2.

4. Materials Science, Brookhaven National Laboratory, Upton, NY 11973, USA.

* Corresponding author: regerton@ualberta.ca

The delocalization of inelastic scattering is described by a point spread function $\operatorname{PSF}(r)$ that represents the scattering probability as a function of the distance $r$ from the trajectory of an incident electron. For core-loss scattering, the PSF has sub-nm or even subatomic dimensions but for low-loss scattering (energy loss $E<50 \mathrm{eV}$ ) its width can be several $\mathrm{nm}$ or tens of $\mathrm{nm}$. This width determines the spatial resolution of TEM-image features that arise from inelastic scattering, and partially determines the minimum line-width achievable in electron-beam lithography [1] or e-beam deposition [2,3].

Quantum dipole theory gives PSF $\sim\left[\mathrm{K}_{1}\left(r / b_{\max }\right)\right]^{2}+\left[\mathrm{K}_{0}\left(r / b_{\max }\right)\right]^{2}$ with $b_{\max }=1 /\left(k_{0} \theta_{\mathrm{E}}\right)$. Fourier transform of the angular distribution of the inelastic scattering amplitude gives PSF $\approx\left[b_{0}{ }^{2} /\left(\mathrm{r}^{2}+b_{0}{ }^{2}\right)\right] \exp \left(-r / b_{\max }\right)$ with $b_{0} \approx 1 /\left(2 k_{0} \theta_{\mathrm{c}}\right)$. Both expressions yield very similar results for $r>b_{0}$ but the second version avoids a singularity at $r=0$ by including a cutoff in the angular distribution of intensity at $\theta_{\mathrm{c}}=\left(2 \theta_{\mathrm{E}}\right)^{1 / 2}$. But doubt remains about the most appropriate value of $b_{0}[4]$, which is perhaps best resolved experimentally.

Measurement of the PSF is possible by recording a sub-nm probe (focused on a thin specimen) through an imaging filter (e.g. Gatan GIF). The specimen should be thin enough to avoid significant beam broadening, and aberrations of the probe-forming and imaging lenses must be minimized [4]. Our procedure has been to focus and aberration-correct the objective and condenser lenses with the GIF set for zero loss, then increase the TEM high voltage by a few $\mathrm{eV}$ and refocus the condenser system for minimum image width (if necessary) before recording the probe image at high magnification.

Results are shown in Figs. 1 and 2. The measured PSF approximates to $1 / r^{2}$ for $r>0.1 \mathrm{~nm}$, as expected. The full width at half maximum (FWHM) exceeds $2 b_{0}$, likely due to a change in phase or incoherency of the inelastic scattering at higher angles [4]. For $E<5 \mathrm{eV}$, the estimated median delocalization diameter is about $60 \%$ of that given by the approximate formula: $d_{50} \approx 16 \mathrm{~nm} / E^{3 / 4}$. For $E>5 \mathrm{eV}$, our measured FWHM and $d_{50}$ values start to increase with increasing energy loss, suggesting that chromatic aberration is interfering with measurement at these higher values of energy loss [5].

\section{References:}

[1] G. Han and F. Cerina, J. Vac. Sci. Technol. B 18 (2000) 3297.

[2] N. Silvis-Cividjian, C.W. Hagen, P. Kruit, J. Appl. Phys. 98 (2005) 084905.

[3] P.A. Crozier, J. Vac. Sci. Technol B 26 (2008) 249.

[4] R.F. Egerton, Microscopy (2018) i52-i59, doi: 10.1093/jmicro/dfx089

[5] The authors acknowledge funding the Natural Sciences and Engineering Research Council of Canada. 

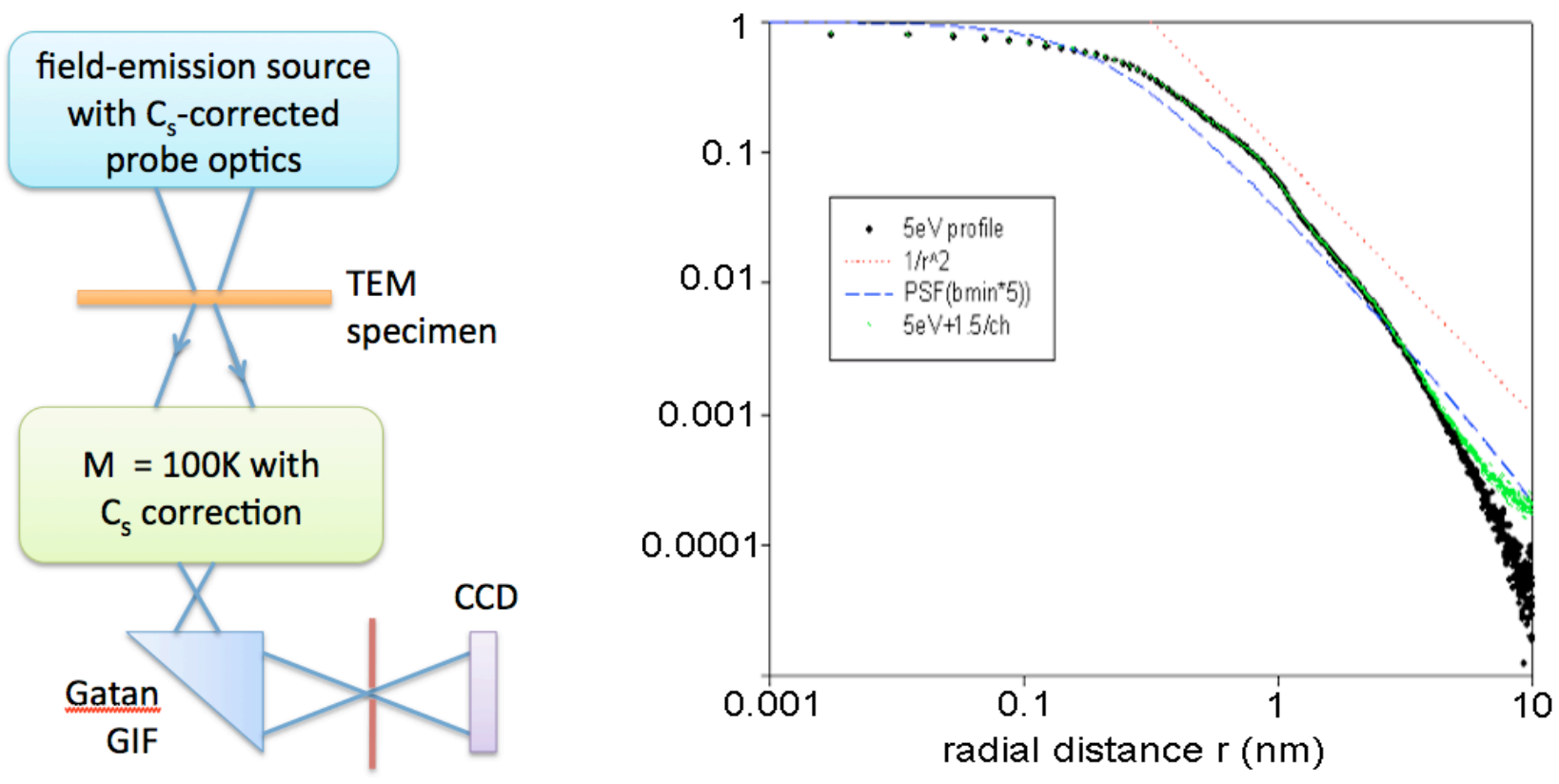

Figure 1. Left: measurement scheme schematic. Right: Inelastic-scattering PSF as measured (black data points) and with background correction (green data points) together with a PSF calculated using the Lorentzian formula (blue dashed curve) and compared with a $1 / r^{2}$ dependence (dotted red line).
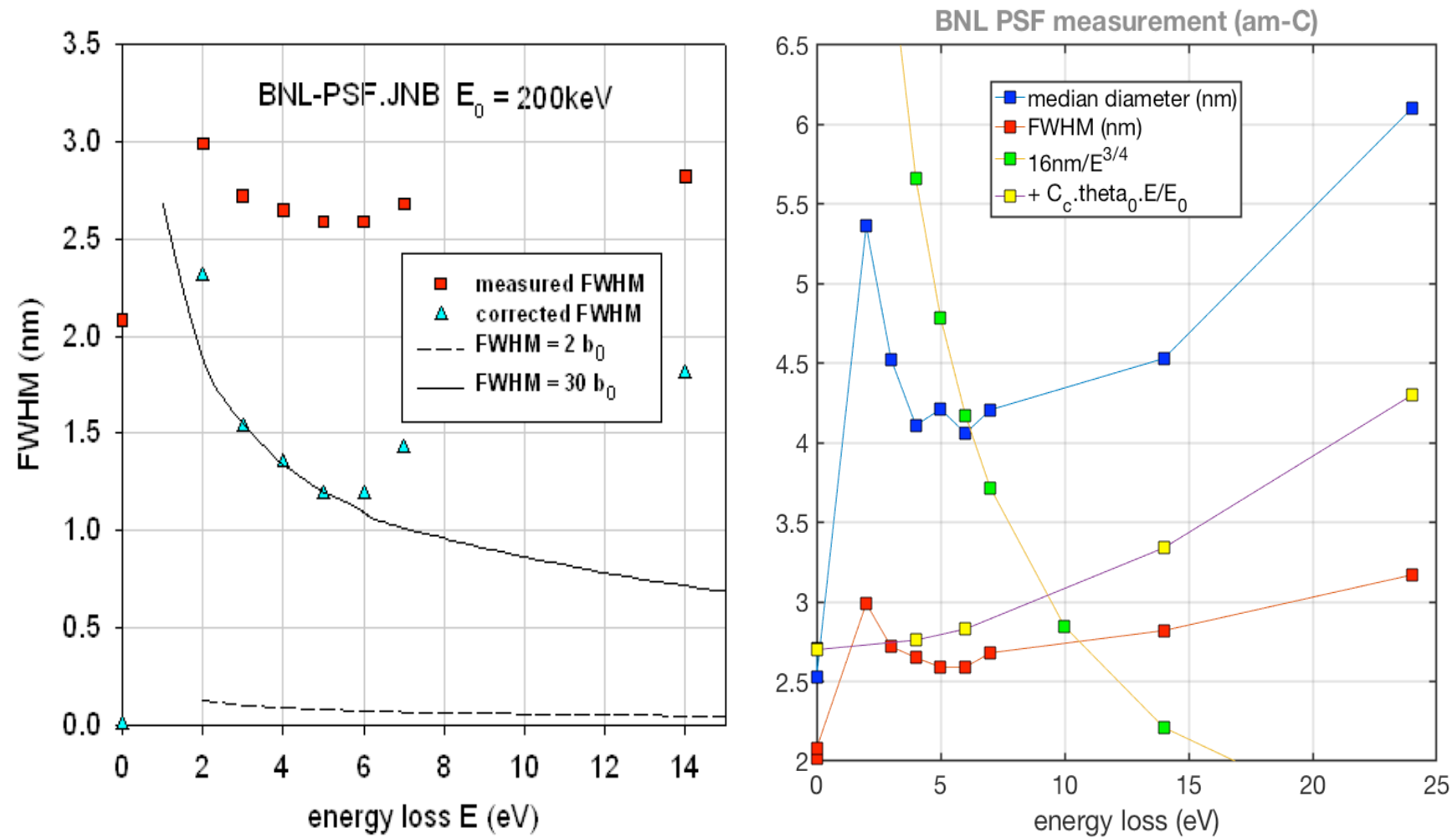

Figure 2. Left: FWHM before and after correcting for $0-\mathrm{eV}$ probe diameter, compared with two estimates based on theory. Right: measured median diameter (blue squares) compared with $16 \mathrm{~nm} / E^{3 / 4}$ (descending curve, green squares). The two lower curves show the measured FWHM (red squares) compared with a schematic estimate of chromatic aberration (yellow data points). 